\title{
Stochastic Approximations of Hybrid Systems
}

\author{
Alessandro Abate, Aaron D. Ames, S. Shankar Sastry \\ Department of Electrical Engineering and Computer Sciences \\ University of California at Berkeley \\ Berkeley, CA 94720 \\ \{aabate, adames, sastry\}@eecs.berkeley.edu
}

\begin{abstract}
This paper introduces a method for approximating the dynamics of deterministic hybrid systems. Within this setting, we shall consider jump conditions that are characterized by spacial guards. After defining proper penalty functions along these deterministic guards, corresponding probabilistic intensities are introduced and the deterministic dynamics are approximated by the stochastic evolution of a continuous-time Markov process. We will illustrate how the definition of the stochastic barriers can avoid ill-posed events such as "grazing," and show how the probabilistic guards can be helpful in addressing the problem of event detection. Furthermore, this method represents a very general technique for handling Zeno phenomena; it provides a universal way to regularize a hybrid system. Simulations will show that the stochastic approximation of a hybrid system is accurate, while being able to handle "pathological cases." Finally, further generalizations of this approach are motivated and discussed.
\end{abstract}

\section{INTRODUCTION}

Recently, a considerable amount of research has been directed towards the topic of Stochastic Hybrid Systems (SHS for short), both in the viewpoint of extending the theory of deterministic HS (cf. [4] and [11]), as well as discovering new applications unique to the probabilistic framework. This, in our view, is due to the increasingly clear limitations of the deterministic setting. The main challenge that the HS framework poses-unlike the case of classical dynamical systems or the relatively simple case of switched systems ${ }^{1}$-is the necessity of handling spacial guards and therefore discrete-time conditions. These jump conditions often give rise to pathological behaviors, like "grazing" or Zeno; their presence prevents the possibility of globally understanding the dynamical properties of a HS, e.g., determining stability or reachability; moreover, they heavily influence the continuity of a hybrid trajectory with respect to its initial condition. The study of Optimal Control for HS is yet another instance of how challenging it is to determine the global behavior of such a system based on its local dynamics. The current state of research in the "deterministic world" has produced results which are either applicable to very special cases, or which require particularly strong conditions on the system's structure; for

\footnotetext{
This research is supported by the National Science Foundation (NSF award number CCR-0225610)

${ }^{1}$ In simple terms, we define switched systems a collection of dynamical systems on the same vector space; the global dynamics jumps between them with conditions in time, which can be either deterministic or probabilistic.
}

example, the fundamental concept of stability for HS has produced only few results, the most notable of which can be found in [3] and [12].

The presence of Zeno behavior in hybrid systems provides another important example of the unique challenges confronted in the theory of deterministic HS. Qualitatively speaking, Zenoness describes a condition where the hybrid trajectory switches between specific domains infinitely many times over a finite time interval. It represents a modeling error which sometimes can be avoided by introducing finite delays in the switches, which in fact means changing the model itself. In a more simplified setting, that of Affine $\mathrm{HS}$, some interesting results have been also obtained in [2].

These are just a few examples of the many aspects of deterministic HS that pose a challenge to their analysis. On the other hand, approximating a HS with a stochastic counterpart can give a fresh new perspective to the problem. In fact, along with being more general than its deterministic counterpart, it could offer, as we shall hint at, some tools to look at the global behavior of HS. In the case of Zeno phenomena for a HS, we shall show that our stochastic approximation is not Zeno; stochastic approximations provide a method for universally regularizing a hybrid system, i.e., they are a de-abstraction of the hybrid model which could be of interest to software developers.

The paper will unfold as follows: after introducing the classical definition of a HS, we will describe how to substitute its guards with probabilistic barriers. A section on discrete event handling follows. Then, we shall focus on Zeno conditions. Simulations will validate our statements. Conclusions will close out the paper.

\section{The Setting}

Throughout the paper we shall stick to the classical framework for deterministic HS [12].

Definition 1: Define a hybrid system as a tuple $\mathscr{H}=$ $(Q, E, D, G, R, F)$ where

- $Q=\{1, \ldots, m\} \subset \mathbb{Z}$ is a finite set of discrete states.

- $E \subset Q \times Q$ is a set of edges which define relations between the domains. For $e=(i, j) \in E$ denote the source of $e$ by $\mathfrak{s}(e)=i$ and the target of $e$ by $\mathfrak{t}(e)=j$.

- $D=\left\{D_{i}\right\}_{i \in Q}$ is a set of domains where $D_{i}$ is a compact subset of $\mathbb{R}^{n}$.

- $G=\left\{G_{e}\right\}_{e \in E}$ is a set of guards, where $G_{e} \subseteq D_{\mathfrak{s}(e)}$; we assume that there exists a collection of smooth 
functions $\left\{g_{e}\right\}_{e \in E}$ such that $G_{e}=\left\{x: g_{e}(x)=0\right\}$; we also assume that $g_{e}(x) \geq 0$ for all $x \in D_{s(e)}$.

- $R=\left\{R_{e}\right\}_{e \in E}$ is a set of reset maps; these are continuous maps from $G_{e} \subseteq D_{\mathfrak{s}(e)}$ to $R_{e}\left(G_{e}\right) \subseteq$ $D_{\mathfrak{t}(e)} \backslash G_{\mathfrak{t}(e)}$.

- $F=\left\{f_{i}\right\}_{i \in Q}$ is a set of vector fields such that $f_{i}$ is Lipschitz on $\mathbb{R}^{n}$. The solution to the ODE $f_{i}$ with initial condition $x_{0} \in D_{i}$ is denoted by $x_{i}(t)$ where $x_{i}\left(t_{0}\right)=x_{0}$.

A execution $^{2}$ of the hybrid system $\mathscr{H}$ is a tuple $\chi=$ $(\tau, \xi, \eta)$ where

- $\tau=\left\{\tau_{i}\right\}_{i \in \mathbb{N}}$ with $\tau_{0}=0 \leq \tau_{1} \leq \cdots \leq \tau_{j} \leq \cdots$ is a hybrid time sequence or a sequence of switching times.

- $\xi=\left\{\xi_{i}\right\}_{i \in \mathbb{N}}$ with $\xi_{i} \in \bigcup_{i \in Q} D_{i}$ is a sequence of initial conditions $^{3}$.

- $\eta=\left\{\eta_{i}\right\}_{i \in \mathbb{N}}$ with $\eta_{i} \in E$ is a hybrid edge sequence.

Additionally, we require that $\chi=(\tau, \xi, \eta)$ must satisfy the condition that for $i \in \mathbb{N}$,

$$
\begin{aligned}
\xi_{i} & =x_{\mathfrak{s}\left(\eta_{i}\right)}\left(\tau_{i}\right) \\
\tau_{i+1} & =\min \left\{t \geq \tau_{i}: x_{\mathfrak{s}\left(\eta_{i}\right)}(t) \in G_{\eta_{i}}\right\} \\
\mathfrak{s}\left(\eta_{i+1}\right) & =\mathfrak{t}\left(\eta_{i}\right) \\
\xi_{i+1} & =R_{\eta_{i}}\left(x_{\mathfrak{s}\left(\eta_{i}\right)}\left(\tau_{i+1}\right)\right)
\end{aligned}
$$

We also require that $\varphi_{t-\tau_{i}}^{\mathfrak{s}\left(\eta_{i}\right)}\left(\xi_{i}\right) \in D_{\mathfrak{s}\left(\eta_{i}\right)}$ for all $t \in$ $\left[\tau_{i}, \tau_{i+1}\right]$; this is quite a natural assumption. With this notation, define a function $q: \tau \rightarrow Q$ by setting $q(t)=$ $\mathfrak{s}\left(\eta_{i}\right)$ if $t \in\left[\tau_{i}, \tau_{i+1}\right]$; we call this function the discrete evolution of the execution $\chi$.

A key point is the understanding that many of the subtleties mentioned in the introduction are due to the presence of the spacial guards; for example, particular configurations of these guards can result in Zeno phenomena; their combination with the reset maps can be accounted for the discontinuity of the hybrid trajectories. The first fact is the main impetus for considering these spacial guards in more detail and for defining intensity functions based on them.

\section{A. Defining Transition Intensities}

Let us consider a set of state-dependent functions, one for each guard of every single domain of the deterministic HS. They are defined on the entirety of the domains:

$$
\begin{aligned}
\lambda: E \times X & \rightarrow \mathbb{R}_{0}^{+}, \\
(e=(i, j), x) & \mapsto \lambda_{i j}(x)
\end{aligned}
$$

for $i=1, \ldots, m$ and $j=1, \ldots, m_{i}$, where $m_{i}$ is the cardinality of the guard set in the domain $D_{i}$. These functions are intended to represent jumping intensities at

\footnotetext{
${ }^{2}$ Here we are considering only infinite executions since these are the executions that display Zeno behavior; introducing the definition of a finite execution would require unnecessary complication. For the more general definition see [12] or [15].

${ }^{3}$ The reader should notice that we include the guards in the initial points set: this is in practice not detrimental for our results.
}

a specific point in the domain, with respect to one of its guards. Intuitively, they will be roughly equal to zero inside a domain, while growing (to infinity) in a neighborhood of the guard for which they are defined.

The intensity functions are defined so that they account for both the distance to the guard, and the relative direction of the trajectory in the domain with the guard. Assume the domain $D_{i}$ is characterized by two elements: the vector field $f_{i}(x)$ and the guard function $g_{i j}(x)$ :

$$
\begin{aligned}
& \dot{x}=f_{i}(x) ; \\
& g_{i j}(x) \geq 0 .
\end{aligned}
$$

It is possible to transform the general, possibly nonlinear guard $g_{i j}(x)$ into a linear one by introducing a new variable $z=g_{i j}(x)^{4}$, and defining the new extended system (cf. [14]):

$$
\begin{aligned}
& \dot{x}=f_{i}(x), \\
& \dot{z}=L_{f_{i}} g_{i j}(x)=\frac{\partial g_{i j}(x)}{\partial x} f_{i}(x) ; \\
& z \geq 0 .
\end{aligned}
$$

The term in the second equation is the Lie derivative of the function $g_{i j}$ along the vector field $f_{i}$. It describes any trajectory of the vector field from the perspective of a guard. The last inequality describes the equivalent guard in the extended domain. We define the jump intensity $\lambda_{i j}(x)$ as:

$$
\lambda_{i j}(x)=\frac{1}{\|z\|} \frac{\dot{z}}{\|\dot{z}\|}=\frac{1}{\left\|g_{i j}(x)\right\|} \frac{L_{f_{i}} g_{i j}(x)}{\left\|L_{f_{i}} g_{i j}(x)\right\|} .
$$

The first term is inversely proportional to the distance from the guard. The second term takes into account the derivative of the guard along the vector field and, by normalization, is a number between zero and one; in the case of linear guards, i.e., guards that are hyperplanes, it can be interpreted as being proportional to the angle between the vector field and the guard; it is zero when the vector field is tangent to the hyperplane, while it is one in the orthogonal case. The motivation to include the second term comes from the need to avoid grazing conditions [5], i.e. flows that osculate the guard, without actually crossing it (see Fig. 1). The reader should ponder over the observation that other possible jump functions could be defined. For instance, the information coming from higher order Lie derivatives could be exploited in the definition; alternatively, the functions could embed the information of whether the vector field points into or out of the domain. It will nevertheless become clear in the following how this will be helpful only from a simulation standpoint, while in theory similar function may be indeed equivalent.

This approach is not new in the literature; the idea of employing space-dependent rates has been recently used in

\footnotetext{
${ }^{4}$ If each domain has a different number of guards, it is necessary to introduce in all of them a number of variables equal to the maximum cardinality of the set of guards among the domains; possibly some of the new variables will describe equilibrium surfaces for the extended domain.
} 


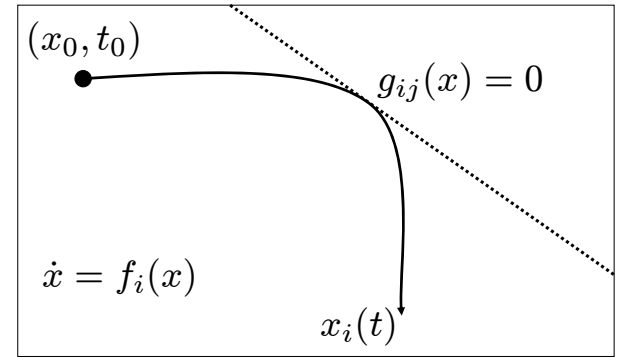

Fig. 1. A grazing condition.

[10]. In this particular instance the author provides a specific intensity function for the "bouncing ball" hybrid system given by:

$$
\lambda_{11}^{\epsilon}(x)=\epsilon e^{-x / \epsilon},
$$

where $\epsilon$ is a constant parameter that tunes the accuracy of the approximation; the indices describe the presence of just one domain and its relative guard. This work did not provide any hint on how to give these intensity functions in generality. Such a shape could be exploited and embedded with our transitions rates.

\section{B. Jump Intensities as a function of time}

Thus far, we have regarded the jump intensities as functions of the state space in each domain. A related idea, that of defining time-dependent jump conditions, has been used in the literature for Markov-jump systems [13], as well as, albeit from another perspective, for continuous-time Markov Chains [9]. In [6], space-dependent jump intensities are defined for piecewise-deterministic Markov processes. To begin with, we regard the execution of a hybrid system as a stochastic process (jump process) on a probability space $(\Omega, \mathcal{F}, \mathbb{P})$. Focusing on a domain $D_{i}$ and considering the guard associated to the edge $e=(i, j)$, we can think of these rates as time-dependent functions when we fix an initial condition $x_{0}$ and consider the evolution in time of the flow of the related vector field from that point: $\forall t \geq t_{0}$, we can consider $\lambda_{i j}\left(x_{i}(t)\right)$. Here the function $x_{i}(t)$ describes the solution of the ordinary differential equation $\dot{x}=f_{i}(x)$ with initial condition $x_{i}\left(t_{0}\right)=x_{0}$.

More generally, consider the collection of domains $\left\{D_{1}, D_{2}, \ldots, D_{m}\right\}$; we can associate to each guard in each domain $D_{i}$ which transitions the hybrid system to the domain $D_{j}$ for $j \neq i$, or equivalently to each edge $e=$ $(i, j)$, a rate $\lambda_{i j}\left(x_{i}(t)\right), \forall t \geq t_{0}$. From this, define an additional rate which will be related to the length of the dwelling time within the domain $D_{i}$ :

$$
\nu_{i}(t)=\sum_{j \neq i} \lambda_{i j}\left(x_{i}(t)\right), \quad \forall i=1, \ldots, m ; \forall t \geq 0 .
$$

Here it is important to mention the possible extensions of the former quantities to guards that are associated with reset maps that may force the solution back into the same domain. In order to maintain the classical properties of the matrix of jumping rates, it will be necessary to introduce a dummy copy of each such domain with same vector fields and guards. We leave to the reader the task of sorting out this simple subtlety.

Assume $\tau_{r}$ is the time of the $r^{\text {th }}$ jump happening in a domain, say, the domain $D_{i}$. Resorting to the theory of continuous-time Markov chains, we know that the probability that the first jump happening in domain $D_{i}$ after time $t$, starting at time $t_{0}$, is

$$
\mathbb{P}_{i}\left(\tau_{r}>t\right)=e^{\int_{t_{0}}^{t}-\nu_{i}(s) d s} .
$$

In other words, the $P_{i}$ distribution of $\tau_{r}$ is exponential with parameter $\nu_{i}(t)$. The reader should note the nonhomogeneity of the process. Considering a differential in time $d t$, the probability to jump from domain $D_{i}$ to domain $D_{j}$ in $[t, t+d t)$ is

$$
\begin{gathered}
\mathbb{P}(q(t+d t)=j \mid q(t)=i ; q(s), s<t)= \\
\lambda_{i j}(t) d t+o(d t), j \neq i,
\end{gathered}
$$

where we have exploited a markovian property for the process $q$. Similarly, the probability that in the interval $[t, t+d t)$ the domain $D_{i}$ would not change is

$$
\begin{gathered}
\mathbb{P}(q(t+d t)=i \mid q(t)=i ; q(s), s<t)= \\
1-\sum_{j \neq i} \lambda_{i j}(t) d t+o(d t)=1-\nu_{i}(t) d t+o(d t) .
\end{gathered}
$$

This can actually be inferred directly from (3) by approximation. At this point the connection between this approach and the literature on (piecewise) continuous-time Markov processes and non-homogeneous Poisson processes should be recognizable.

Remark 1: The reader should note how this approach handles one of the fundamental intricacies of HS, that is the relative position and possible intersection between the guards in each domain. As a matter of fact, if there is more than one guard per single domain, the jump intensity functions will be endowed with the property of being composable.

\section{Stochastic Approximations for Event DETECTION}

Numerical simulation is an important tool for the analysis of HS; nevertheless, it is well known that it is rather brittle with respect to initial conditions. Small changes in the initial condition of a hybrid trajectory do not necessarily result in small changes in its final position. Moreover, the possible failure to detect an event that happens for the actual solution by considering the evolution of the numerical trajectory may result in dynamics that are qualitatively different from those of the actual trajectory. Some notorious examples of mishandled events are summarized in Fig. 2.

The simulation process heavily relies on approximation techniques for integrating the vector fields on each domain (cf. [7]). In this work we claim that, given a numerical technique to integrate the ODE's in each domain, the stochastic approximation of the guard functions can be an 


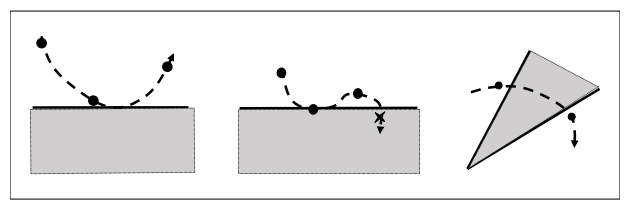

Fig. 2. In some cases the event is either not detected or even wrongly spotted. The shaded regions are not parts of the domain and their marked margin represents the guard.

effective framework for handling discrete events. This claim is supported by the observation that the stochastic guard functions represent a special kind of "barrier functions" that can be related to the flow of the system. As described in the first section of the paper, we can make the jump condition depend on the actual magnitude of the function given in Eqn. (1), rather than on the simple examination of the value of the guard $g(x(t))$; it should be clear how the former condition is stronger and more discriminative than the latter. Moreover, as already pointed out, the barrier functions are endowed with a term that prevent discrete switching in case when the trajectory is parallel to the guard-this prevents grazing.

Additionally, in order to avoid the necessity of setting the integration step-size a priori, we propose a dynamic refinement of it, depending on the value of the stochastic boundary. Similar in concept as what was suggested in [8], this idea can be implemented on a variable-step-size integration method.

Consider the solution $x(t)$ to the differential equation $\dot{x}=$ $f(x)$ with $x\left(t_{0}\right)=x_{0}$. Assume it is approximated at the discrete time sequence $\left\{t_{k}\right\}_{k=0}^{l}$, for $t_{k} \geq t_{k-1}$, as $\hat{x}_{k}=$ $\hat{x}\left(t_{k}\right)$. The value of the vector field at that time is then $f_{k}=f\left(\hat{x}_{k}\right) \forall k=1, \ldots, m$. The time step used in the numerical integration is defined as $h_{k}=t_{k}-t_{k-1}$. A simple integration scheme that employs $m$ past values of the vector fields can be described by the following difference equation

$$
x_{k+1}=x_{k}+h_{k+1} \sum_{j=1}^{m} \beta_{j} f_{k-j+1},
$$

where the coefficients $\beta_{i}$ are functions of the $m$ previous steps and can be determined through evaluation, as can be verified in [7]. As in [8], assume that the step number $k$ lies in the continuum, $k \in \mathbb{R}_{0}^{+}$, and time to be a function of it, $t=t(k)$. The stochastic guard function relating to the function $g(x)$ can then be described along a trajectory by $\lambda[g(x(t))]=\lambda\{g[x(t(k))]\}$. The step size of the integration method can be regarded as $h(k)=\frac{d t}{d k}$; we should keep in mind that we can control it. Then, we can look at how the barrier function varies with respect to the (continuous) step number:

$$
\frac{d \lambda}{d k}=\frac{\partial \lambda}{\partial g} \frac{\partial g}{\partial x} \frac{\partial x}{\partial t} \frac{\partial t}{\partial k}=\frac{\partial \lambda}{\partial g} L_{f} g(x) h(k)=L_{f} \lambda(x) h(k) .
$$

Now, if we regard the term $h(k)$ as a control input, we can choose its shape, in order to obtain the desired dynamics.

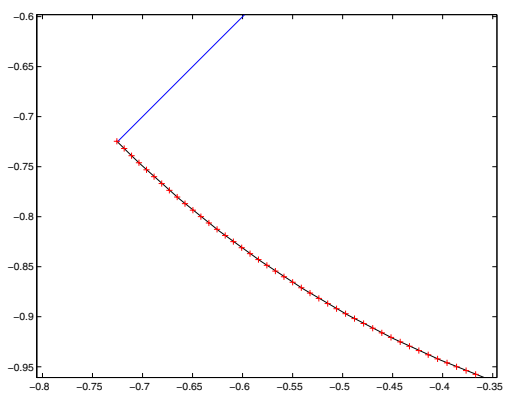

Fig. 3. Event detection; the continuous straight line represents the guard; the other continuous curve depicts the deterministic trajectory, to which a classical event detection method is applied. The crosses tell where the integration points fall in the stochastic framework.

More precisely, if we make it a nonlinear function of the state and the barrier function as follows

$$
h(k)=\frac{\alpha}{\left(L_{f} \lambda(x)\right) \lambda(x)}, \quad \alpha>0,
$$

then we get

$$
\frac{d \lambda(k)}{d k}=\frac{\alpha}{\lambda(k)} ; \quad \lambda(0)=\lambda_{0} .
$$

The solution of this differential equation tells us that if the of the vector field approaches the guard, the boundary function $\lambda(x)$ diverges; moreover, in this instance the step size is dynamically decreased to zero. In other words, the closer we get to the guard, the more precise the integration step will be and we will "force" the integration of the differential equation to detect the event before crossing the guard.

We present the results of two simulations. The first one (see Fig. 3), shows how the stochastic approximation precisely detects the crossing, as compared to the deterministic numerical method. In this instance the vector field is a circular one, rotating clockwise. The guard is represented by the line, slanted at $\pi / 4$ degrees.

The second simulation, which can be seen in Fig. 4, shows how, given a numerical solution that actually grazes the guard, the stochastic approximation finely avoids to detect the event. Again, in this example the vector field rotates clockwise, while the guard is slanted at $-\pi / 4$ degrees.

\section{Handling Deterministic Zeno Dynamics}

Hybrid Systems pose a problem which is unknown in the simpler setting of dynamical systems, that of Zeno dynamics. In simple terms, Zeno dynamics describe the condition when, in a finite time interval, the hybrid trajectory jumps between specific domains infinitely many times. More precisely, consider the following definition.

Definition 2: A hybrid system $\mathscr{H}$ is Zeno if for some execution $\chi$ of $\mathscr{H}$ there exists a finite constant $\tau_{\infty}$ (called the Zeno time $)$ such that $\lim _{i \rightarrow \infty} \tau_{i}=\sum_{i=0}^{\infty}\left(\tau_{i+1}-\tau_{i}\right)=$ $\tau_{\infty}$. the execution $\chi$ is called a Zeno execution. 


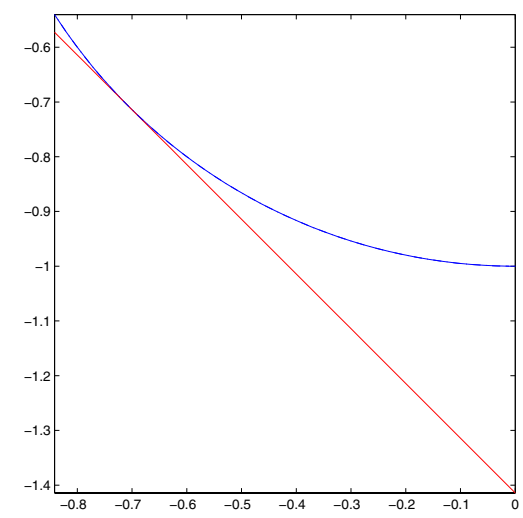

Fig. 4. In the case of grazing, the stochastic approximation perfectly matches the numerical solution (curved lines) and does not detect the event determined by the presence of the guard (straight line).

The definition of a Zeno execution results in two qualitatively different types of Zeno behavior. They are defined as follows: for an execution $\chi$ that is Zeno, $\chi$ is

Chattering Zeno: If there exists a finite $C$ such that $\tau_{i+1}-\tau_{i}=0$ for all $i \geq C$.

Genuinely Zeno: If $\tau_{i+1}-\tau_{i}>0$ for all $i \in \mathbb{N}$.

The difference between these is especially prevalent in their detection and elimination. Genuinely Zeno executions are much more complicated in their behavior. This paper will focus on genuine Zeno. There have been many attempts to handle the Zeno behavior in a deterministic setting by either changing the original model [15] with the addition of some delays, or looking at it within a simpler setting [2]. Hespanha, [10], first suggested that, in a very special case, a stochastic sequence of approximated HS could effectivly handle Zeno behavior.

The definition of our probabilistic barriers have this property in a general case, given some additional structural assumptions. Frist, the reader should note that:

Remark 2: The definition of the rates $\lambda_{i j}(x), \forall i, j$, implies their measurability $\forall x \in D_{i}$.

Moreover, we shall assume the following:

Assumption 1: The jumping rates $\lambda_{i j}$ are locally integrable, $\forall i, j$. A sufficient condition would be, for example, their boundedness.

Remark 3: The local integrability condition, while it may appear restrictive from a theoretical point of view, is always valid when a real simulation of the HS is preformed.

The following holds:

Theorem 1: Let $\mathscr{H}$ be a hybrid system and $\mathscr{S}$ be its stochastic approximation. The set of trajectories of $\mathscr{S}$ that are Zeno has measure zero. In other terms, $\mathscr{S}$ is Zeno with probability zero.

Proof: Consider a hybrid time stochastic sequence $\tilde{\tau}=$ $\left\{\tilde{\tau}_{i}\right\}_{i \in \mathbb{N}}$. Reason by contradiction: assume the SHS $\mathscr{S}$ is
Zeno. Then, with probability one, there would exist a finite constant $\tilde{\tau}_{\infty}<\infty$ such that $\lim _{i \rightarrow \infty} \tilde{\tau}_{i}=\sum_{i=0}^{\infty}\left(\tilde{\tau}_{i+1}-\right.$ $\left.\tilde{\tau}_{i}\right)=\tilde{\tau}_{\infty}$. Assume for the moment that, $\forall i \in Q, \nu_{i}(t)=$ $\nu(t), \forall t \geq t_{0}$. Considering a single time interval $\left[\tilde{\tau}_{i}, \tilde{\tau}_{i+1}\right)$ and a random variable $J$ that is the time at which a discrete event occurs. The probability that the jump happens inside this interval is

$$
\mathbb{P}\left(J \in\left[\tilde{\tau}_{i+1}, \tilde{\tau}_{i}\right)\right)=1-e^{-\int_{\tilde{\tau}_{i}}^{\tilde{\tau}_{i+1}} \nu(s) d s} .
$$

Exploiting the assumption of local integrability and observing that the interval is always finite, the probability of the previous event is always less than one. Therefore, by construction the SHS $\mathscr{S}$ cannot be Zeno in probability.

Let us show now that the probability of this event to happen is actually zero. Given a stochastic Zeno time sequence $\tilde{\tau}=\left\{\tilde{\tau}_{i}\right\}_{i \in \mathbb{N}}$, identify with it the infinite subsequence that contains jumps at each subinterval: $\tilde{\tau}^{*}=\left\{\tilde{\tau}_{i}^{*}\right\}_{i \in \Lambda \subseteq \mathbb{N}}$. Call $\tilde{\tau}^{* c}=\left\{\tilde{\tau}_{i}^{* c}\right\}_{i \in \mathbb{N} \backslash \Lambda}$ the complement of $\tilde{\tau}^{*}$ in $\tilde{\tau}$. It is again a subsequence, possibly infinite, and contains no jumps in all of its subintervals. The probability that $\tilde{\tau}$ exists is then

$$
\begin{aligned}
& \mathbb{P}(\tilde{\tau} \text { exists }) \\
& =\prod_{i \in \mathbb{N} \backslash \Lambda} e^{-\int_{\tilde{\tau}_{i}^{* c}}^{\tilde{\tau}_{i+1}^{* c}} \nu(s) d s} \prod_{i \in \Lambda}\left(1-e^{\left.-\int_{\tilde{\tau}_{i}^{*}}^{\tilde{\tau}_{i+1}^{*} \nu(s) d s}\right)}\right. \\
& =e^{-\sum_{i \in \mathbb{N} \backslash \Lambda} \int_{\tilde{\tau}_{i}^{* c}}^{\tilde{\tau}_{i c}^{* c}} \nu(s) d s} \prod_{i \in \Lambda}\left(1-e^{-\int_{\tilde{\tau}_{i}^{*}}^{\tilde{\tau}_{*}^{*}} \nu(s) d s}\right) .
\end{aligned}
$$

Recall the assumption that the function $\nu(s)$ is measurable. The first term is always less than one (being equal to one only in case of an infinite sequence with infinite cumulative length, which we exclude by assumption). The second term will instead always be equal to zero. In case of Chattering Zeno, the reason for this is trivial. In case of Genuine Zeno, this will also be the case, as the probability is a product of terms less than one decreasing to zero. All in all, the stochastic hybrid time sequence is Zeno on a measure zero set.

If, in general, $\nu_{i}(t) \neq \nu_{j}(t)$, for some $i, j \in Q$, then take $\nu(t)=\sup _{i \in Q} \nu_{i}(t)<\infty, \forall t \geq t_{0}$. Then, the two probabilities above expressed in the general case will be respectively upper bounded by those defined through $\nu(t)$. Thus, also in the general case, Zeno has probability zero to occur for the SHS $\mathscr{S}$.

Building upon the above theorem, we introduce the following fact:

Corollary 1: Let $\mathscr{H}$ be a hybrid system and $\mathscr{S}$ be its stochastic approximation. For any initial point in time $t_{0} \geq$ 0 and initial condition $x_{0} \in D_{i}, i \in 1,2, \ldots, m$, the hybrid trajectory of the SHS $\mathscr{S}$ is globally defined $\forall t \geq t_{0}$.

Proof: We assume that the condition of existence and uniqueness for the hybrid trajectories are satisfied by the definition of the HS $\mathscr{H}$; we implicitly rule out the possibility of blocking conditions, that is the stopping of the trajectory because some terms required for its realization are 


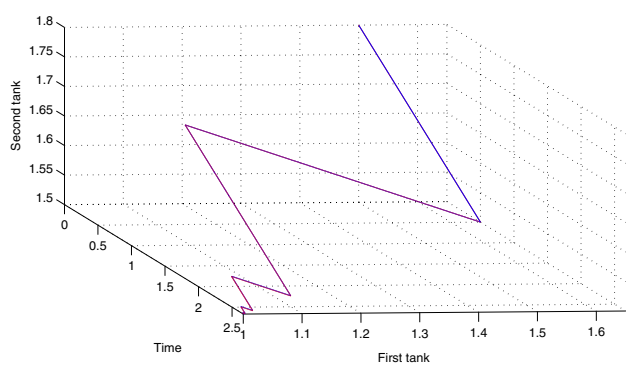

Fig. 5. Three dimensional view of the two water tanks hybrid system.
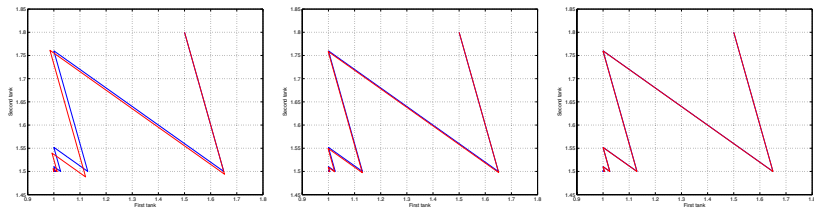

Fig. 6. Top view of the two water tanks hybrid system. We have first simulated this system with the classical deterministic event detection software. After approximating it via the stochastic barrier functions, we have run the simulations with increasing precision.

missing. Moreover, excluding a priori any Zeno behavior, as from the above Theorem, we get to the desired statement.

At this point an example is in order; we worked out a simulation for the well known two water tanks hybrid system (cf. [15]). In this instance, two tanks which present some outflow are filled up with a single hose, which is forced to switch into any one tank which level goes below a certain fixed one. The classical deterministic mathematical model of this HS does not account for delays in the physical switch from one tank to the other tank. This fact accounts for the existance of Zeno behavior. More specifically, this happen in the case when the inflow is less than or equal to the sum of the two outflows. In this case, it is possible to calculate explicitly the exact value of time when this occurs (Zeno time). In Fig. 5, we plotted first a 3D simulation of this system-under conditions that force the system to be Zeno-with the classic deterministic event detection. As expected, the trajectory gets stuck near the Zeno time.

In the series of plots in Fig. 6, we instead show how the stochastic approximation is able to detect the discrete event with as much accuracy than the deterministic case. However, unlike this case, the trajectories are defined for any time; in other words, there is no Zeno time, and the simulation does not stop. In this figure, we have increased the accuracy via a multiplicative parameter in the definition of the barrier function.

\section{FUtURE DIRECTIONS}

The idea of approximating the structure (and thus the dynamics) of a deterministic HS with some stochastic correspondent has far more general consequences than the simple avoidance of Zeno behavior. Handling a probabilistic HS would enable to perform robust analysis on the original
HS. Furthermore, some properties for the original HS may be checked "in probability," rather than in a "worst-case" fashion. Currently, this research is directed towards understanding how the stochastic framework for hybrid systems can be exploited to understand the behavior of the original HS, e.g., stability (cf. [1]) and optimal control.

\section{CONCLUSIONS}

In this work we have proposed a new way to approximate deterministic hybrid systems by substituting their spacial guards, which define the discrete switching conditions, with probabilistic barrier functions. This way, we consider the original HS as a non-homogeneous Markov jump system. The barrier functions are viewed as switching rates in space, but can also be regarded as jump intensities in time along trajectories of the system. We showed how these barriers can be assist in understanding discrete events, with no drawbacks compared the known deterministic methods; the integration step in a simulation can be dynamically changed and adapted, depending on the relative position to the guard, in order to precisely detect the event. Furthermore, we showed how to approximately handle Zeno conditions through this stochastic framework. We provided motivational and applicative simulations showing the features of the methodology, and reflected on the generality of the possible extensions.

\section{REFERENCES}

[1] A. Abate, L. Shi, S. Simic, and S. Sastry, "A stability criterion for stochastic hybrid systems," Mathematical theory of Networks and Systems Conference, Leuwen, BG, July 2004.

[2] A. D. Ames and S. Sastry, "Blowing up affine hybrid systems," 43rd Conference on Decision and Control, Dec 2004.

[3] M. Branicky, "Stability of hybrid systems: State of the art," 36th Conference on Decision and Control, Dec 1997.

[4] M. Bujorianu and J. Lygeros, "Theoretical foundations of stochastic hybrid systems," Mathematical theory of Networks and Systems Conference, Leuwen, BG, July 2004.

[5] P. Collins, "A trajectory-space approach to hybrid systems," Mathematical theory of Networks and Systems Conference, Leuwen, BG, July 2004.

[6] M. Davis, Markov Models and Optimization. Chapman and Hall, 1993.

[7] G. Dshlquist and A. Bjorck, Numerical Methods. Prentice Hall, 1974.

[8] J. M. Esposito, V. Kumar, and G. J. Pappas, "Accurate event detection for simulating hybrid systems," HSCC 2001, LNCS 2033, pp 204217, Apr 2001.

[9] R. Gallager, Discrete Stochastic Processes. Kluwer Academic Publishers, 1996.

[10] J. Hespanha, "Stochastic hybrid sysetms: Application to communication networks," HSCC 2004, LNCS 2993, pp 387-401, Apr 2004.

[11] J. Lygeros, J. Hu, , and S. Sastry, "Towards a theory of stochastic hybrid systems," HSCC 3rd International Workshop, 2000.

[12] J. Lygeros, K. Johansson, S. Simic, J. Zhang, and S. Sastry, Eds., Dynamical Properties of Hybrid Automata, vol. 48, no. 2-18. IEEE, 2003.

[13] M. Mariton, Jump Linear Systems in Automatic Control. Marcel Dekker Inc., New York, 1990.

[14] L. F. Shampine and I. Gladwell, "Reliable solution of special event location problems for odes," ACM Trans. Math. Software, vol. 17, no. 1, pp. 11-25, 1991.

[15] J. Zhang, K. H. Johansson, J. Lygeros, , and S. Sastry, "Zeno hybrid systems," International Journal of Robust and NonLinear Control, vol. 11, pp. 435-451, 2001 